\title{
Determination of wheel/rail contact points in the simulation of railway vehicle dynamics
}

\author{
J. Auciello, S. Falomi, M. Malvezzi, E. Meli \& P. Toni \\ Department of Energetics 'S. Stecco', University of Florence, Italy
}

\begin{abstract}
The numerical simulation of system dynamics is today a standard in the design of railway vehicles; their typical applications are the suspension kinematics, handling performance and ride comfort as well as the generation of load data for lifetime prediction. One of the key points in this type of simulation is the model of the wheel/rail interaction, in other words the definition of the forces exchanged between the wheels and the rail in the contact points. The direction and the magnitude of the contact forces depend on the number and the location of the contact points. The procedure that allows one to define the geometry of the contact then has a significant effect on the reliability of the simulation. The component of the normal contact force to the contact surfaces can be defined as a function of the relative indentation between the surfaces. The component of the contact force tangent to the contact surfaces depends on the relative speeds between the surfaces in the contact area (wheel sliding). The authors have been working on the definition of efficient and reliable models of the interactions between the wheels and the rails and in particular for the definition of the contact points. Different algorithms have been analyzed and compared, based on semi analytical approaches and on neural networks. The paper will summarize the proposed methods and the results obtained from the simulation of two different sceneries. Two different models have been used in this test: the first one was realized with a commercial software, while the second one was developed and implemented by the authors. There is a global agreement between the models, although some differences can be seen during the transients due to the different methods for the determination of the contact points for the integration.
\end{abstract}

Keywords: multibody simulation, wheel-rail contact, real time simulations. 


\section{Introduction}

The numerical simulation of system dynamics is today a standard in the design of railway vehicles. The multibody analysis is applied, for example, to suspension kinematics and compliant kinematics, handling performance and ride comfort as well as to the generation of load data for lifetime prediction. These simulation tasks are usually carried out as off-line simulations performed by means of commercial or customized software.

In recent years Hardware in-the-Loop (HIL) simulation has been extensively introduced in the design of vehicle control systems and the testing of electronic control units, both in the automotive and in the railway fields. The reliability of this type of test is strictly related to the properties of the numerical model that simulates the condition that the tested device would meet in real operative conditions. The numerical model is a representation of the dynamics of the system in which the tested unit is inserted (single vehicle, entire train etc.). In order to obtain reliable results on this type of test rig, the availability of a realistic real time model of the system dynamics is necessary.

Over recent years the MBS (Multibody Simulation) method has then been established in the real-time simulation domain, typically for the design of vehicle control systems and the testing of electronic control units $[1,2]$.

The numerical model described in this paper reproduces the complete threedimensional dynamics of a railway vehicle running on a generic track. It has been developed with the objective of a real time implementation, in order use its results to control the actuators of HIL test rigs.

One of the key points in this study was the definition of a reliable and efficient model describing the forces arising in the wheel/rail interaction areas. The contact force vector is composed of a normal component, resulting from the weight of the vehicle, and by a tangential component resulting from the friction between the wheel and rail in the contact area. This component, named creep force, arises in the contact area when a traction or a braking torque is applied to the axle. These forces play an important role in the wheelset dynamics and their value depends on a number of parameters, including the position of the contact point between the bodies.

The introduction of a well-defined full three-dimensional wheel/rail interaction module in a standard multibody model is not easy and may sensibly increase the complexity and the computational burden of the numerical procedure [3-10].

In the model described in the following sections the wheelset is assumed to have six degrees of freedom with respect to the rails. The local deformation of the contact surface at the contact point is allowed and the normal contact forces are defined using Hertz's contact theory or in terms of assumed stiffness and damping coefficients. This type of approach allows the separation between the wheel and the rail and to manage multiple contact points. One of the main problems correlated with this approach is the definition of the contact point location on line. In most elastic force models, the three-dimensional contact 
problem is reduced, for the sake of efficiency, to a two-dimensional problem when the location of the contact points is searched for.

In order to find a solution for the problem of contact mechanics, detailed descriptions of the surfaces in contact, as well as the kinematics of the bodies, are required. Because wheel and the rail profiled surfaces, the prediction of the location of the contact point on line is a not easy task, especially when the most general three-dimensional motion of a wheelset with respect to the rails is considered. In many cases, some simplifications are made in the geometric and/or the kinematic description. A common method used in many existing computer algorithms to find the location of the contact point is to interpolate some precalculated table entries. The location of the contact point is given as function of some coordinates that measure the relative position of the wheelset with respect to the rails. The degree of accuracy of such algorithms depends on the number of coordinates that are used to define this relative position.

The developed numerical procedure can be schematized as shown in Figure 1. The dynamics of each wheelset depends on the external actions (traction/braking torques) and on the contact forces. The solution of the differential equations describing the wheelset dynamics allows one to calculate the kinematical parameters (displacement and rotation) from which the position of the contact points can be identified. The creep forces are then calculated taking into account the position of the contact points and the wheel creepages, obtained from the wheelset kinematics.

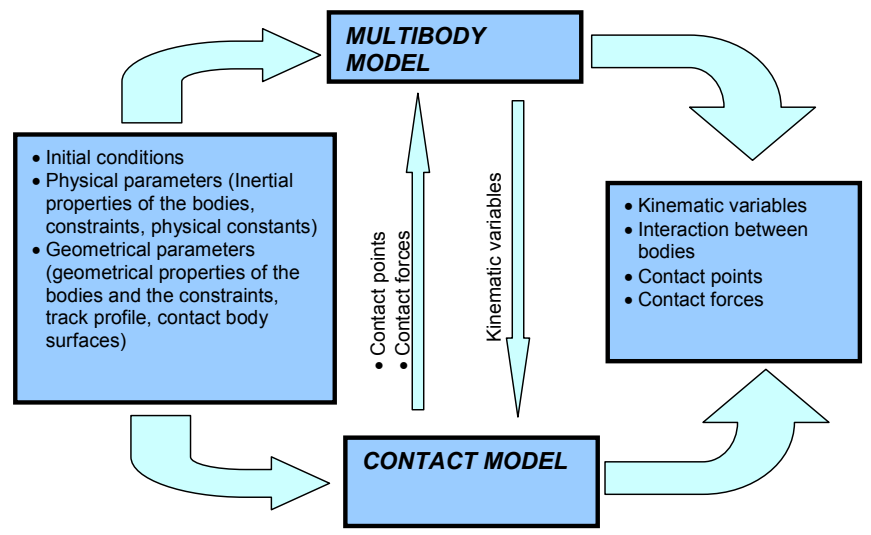

Figure 1: Numerical procedure block diagram.

\section{Definition of wheel/rail contact points}

In Figure 2 the reference frames used to describe the wheel and rail profile geometries are shown. Concerning the rail coordinate system, $x_{b}$ is parallel to the track direction, $y_{b}$ is on the plane defined by the rails and normal to $x_{b}, z_{b}$ 
is consequently defined. The origin $\mathbf{O}_{b}$ is fixed on the track axis, in other words it is on the plane identified by the rails and is equally distant from both of them. The simulated vehicle can move on a generic track, defined by $\gamma(s)$, representing a generic three-dimensional curve defined by means of its parameter $s$. A second auxiliary coordinate system relative to the track is defined, its origin $O_{b^{\prime}}$ is on the median railway curve $\gamma(s(t))$, it is not fixed, and depends on the wheelset motion on the track. The axis $x_{b^{\prime}}$ is tangent to the track curve, the $z_{b^{\prime}}$ axis is normal to the rail plane and $y_{b^{\prime}}$ is consequently defined.

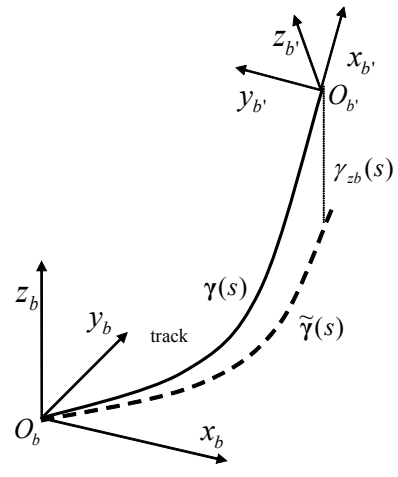

a)

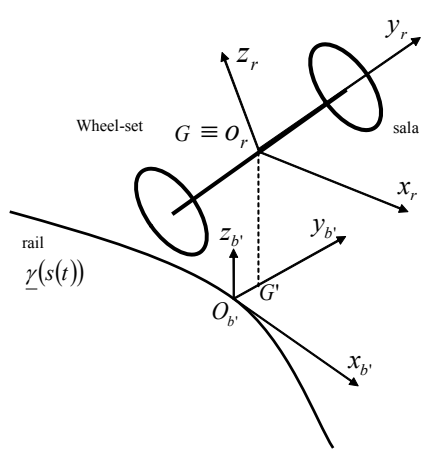

b)

Figure 2: a) Rail coordinate system, b) wheel coordinate system.

The origin $O_{r}$ of the wheelset coordinate system is on the plane $y_{b^{\prime}} z_{b^{\prime}}$, then:

$$
\left[O_{r}(t)-O_{b^{\prime}}(s(t))\right] \bullet \underline{\gamma^{\prime}}(s(t))=0
$$

It is then evident that for any $t$ value, $s(t)$ and then $O_{b}$, can be calculated from the position of the wheelset center of mass.

The wheel is a revolution surface, the wheel radius depends on the $y_{r}$ coordinate, the function $s=s\left(y_{r}\right)$ describes the wheel profile in the $y_{r} z_{r}$ plane, and the coordinates of a generic point on the wheel surface can be expressed, in the wheelset coordinate system, as follows:

$$
\left(P_{r}-O_{r}\right)_{r}=\mathbf{s}_{r}\left(u, y_{r}\right)=\left(\begin{array}{c}
-s\left(y_{r}\right) \sin u \\
y_{r} \\
s\left(y_{r}\right) \cos u
\end{array}\right)
$$


where $u$ is the angle between the radiuses that identify the generic point on the wheel and $-z_{r}$ direction.

The rail surface is an extrusion surface, the function $b=b\left(y_{b^{\prime}}\right)$ describes the rail profile in the plane $y_{b}, z_{b}$, and then the coordinates of a generic point on the rail surface can be expressed as:

$$
\left(P_{b^{\prime}}-O_{b^{\prime}}\right)_{b^{\prime}}=\left(\begin{array}{c}
x_{b^{\prime}} \\
y_{b^{\prime}} \\
b\left(y_{b^{\prime}}\right)
\end{array}\right)
$$

the coordinates of a generic point on the wheel can then be expressed in the auxiliary coordinate system:

$$
\left(P_{b^{\prime}}^{r}-O_{b^{\prime}}\right)=\left(O_{r}-O_{b^{\prime}}\right)_{b^{\prime}}+\left[\mathbf{R}_{2}\right] \cdot O_{r}
$$

The displacement $\left(O_{r}-O_{b^{\prime}}\right)$ and the rotation matrix $\left[\mathrm{R}_{2}\right]$ between the wheelset and the track reference system depend on the relative displacement between the wheelset and the rail, then, for each wheelset configuration the wheel surface can be expressed with respect to the auxiliary reference frame as a function of the parameters $u$ and $y_{r}$ :

$$
P_{b^{\prime}}^{r}=\mathbf{s}_{b^{\prime}}^{r}\left(u, y_{r}\right)
$$

The point $P_{b^{\prime}}$ corresponding to $P_{b^{\prime}}^{r}$ on the rail surface is then given by:

$$
P_{b^{\prime}}=\left(\begin{array}{c}
x_{b^{\prime}}^{r}\left(u, y_{r}\right) \\
y_{b^{\prime}}^{r}\left(u, y_{r}\right) \\
b\left(y_{b^{\prime}}^{r}\right)
\end{array}\right)
$$

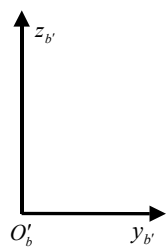

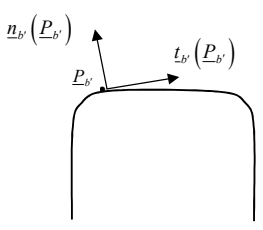

a)

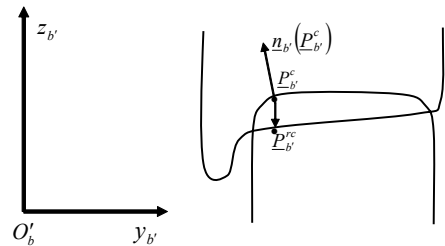

b)

Figure 3: a) Rail surface, normal and tangent directions, b) definition of the difference surface.

The difference surface is then defined as:

$$
D\left(u, y_{r}\right)=\left(P_{b^{\prime}}^{r}-P_{b^{\prime}}\right) \bullet \mathbf{k}_{b^{\prime}}
$$


The contact points are located in the points in which this surface assumes a local minimum. Then, in order to find the contact points the local minima of this surface are searched.

$$
\left\{\begin{array}{c}
\min _{u, y_{r}} D\left(u, y_{r}\right) \rightarrow\left(u_{i}{ }^{c}, y_{i, r}{ }^{c}\right) \\
i=1,2, . ., n \leq 4
\end{array}\right.
$$

The minima are calculated by a procedure that analytically reduces the problem dimension to a single equation that is numerically solved. This method is allows a good precision with low computational loads.

\section{Contact forces between wheel and rail}

Once the local minima have been identified, the indentation between the surfaces is conventionally defined in the direction normal to the rail:

$$
p=\left(P_{b^{\prime}}^{r c}-P_{b^{\prime}}^{c}\right) \bullet \mathbf{n}_{b^{\prime}}\left(P_{b^{\prime}}^{c}\right)
$$

Where $\mathbf{n}_{b^{\prime}}$ is the unitary vector normal to the rail in the contact point. The indentation value is used to calculate the normal component of the contact force, according to the elasto-viscous approach as sum of a term that depends on the normal penetration between the body (the elastic component), and a term proportional to the surface relative velocities in the contact point (the viscous term).

The magnitude of the tangential component of the contact forces is calculated on the basis of Kalker and Hertz theory [12,13]. The Hertz's theory is used to define the contact area dimensions and shape, which depend on the normal force magnitude, the material properties and the local profile geometry. The Kalker linear theory results are used to define the components of the creep forces as follows:

$$
\begin{aligned}
& T_{x}=-f_{11} \xi \\
& T_{y}=-f_{22} \eta-f_{23} \varphi \\
& M_{s p}=f_{23} \eta-f_{33} \varphi
\end{aligned}
$$

where $f_{11}, f_{22}, f_{33}, f_{23}$ are the linear creep coefficients, depending on the contact ellipse semi-axis and on the combined modulus of rigidity (their values are tabulated)[10]; $\xi, \eta$ and $\varphi$ are the values of the creepage components.

\section{Vehicle multibody model}

The multibody model is composed of seven rigid bodies (the car body, two bogies, four wheelsets) connected by three-dimensional non linear elastic- 
viscous force elements modeling the connection elements between the bodies (for example the vehicle suspensions, dampers etc.).

On each wheelset the following force act:

- the creep forces in the contact area;

- the forces due to the interaction with the boogie;

- the external applied braking or traction torque;

- the weight.

The numerical model was realized in the Matlab/Simulink environment, which allows one to obtain a numerically efficient model, to test different types of integration algorithms, to manage singularities. The structure of the model is modular so different subsystems can be easily modeled and substituted in the main procedure. In this type of design environment different and complex systems can be modeled (electrical, pneumatic etc.), while a toolbox devoted to the multibody simulation allows to easily model mechanical systems with a high number of bodies and constraints.

The results of the simulations performed with the developed model were compared with those obtained with models of the same vehicle realized with Adams Rail and Simpack. The benchmark case is the Manchester wagon, whose features are known in the literature and whose Adams Rail model is available [14]. The validation process is necessary to evaluate the accuracy of the model and to verify the performance of the model in terms of computational burden, even if they are obtained using different software environments and different modeling approaches. The differences are particularly evident in the definition of the contact points and of the contact forces.

\section{Results}

In this section some the results obtained from the comparison with the Adams Rail model are briefly described. In tests the vehicle travels along a curve with a constant speed. The parameters that are varied in the first set of tests were:

- $\quad$ the curve radius values (500, 1200 and $2400 \mathrm{~m})$;

- the rail angle was $1 / 40$ and $1 / 20$;

- $\quad$ the cant angles were 40/1435 rad and 90/1435 rad;

- the speed values were 15,30 and $45 \mathrm{~m} / \mathrm{s}$.

The wheel/rail adhesion coefficient was 0.2 in all the tests. In this set of tests the displacements of the wheelset centers and the contact forces were analyzed and compared.

Figure 4 shows the parameters relative to one of the performed tests: the track curvature and the cant angle. Figure 5 shows the comparison between the results obtained by the Adams and Simulink multibody models, the time-history of the wheelset center of mass displacements is analyzed in the presented example. The results show a good agreement between the different models, a difference between the simulations can be noted in the transient, it is due to the differences in the contact force calculation.

Table 1 summarizes the hunting frequencies calculated through simulations performed imposing different vehicle speeds, a quite good agreement between 
the results obtained with the Simulink and Adams models can be observed. The calculated critical speeds are $71 \mathrm{~m} / \mathrm{s}$ with the Simulink model and $73 \mathrm{~m} / \mathrm{s}$ with the Adams Rail model.

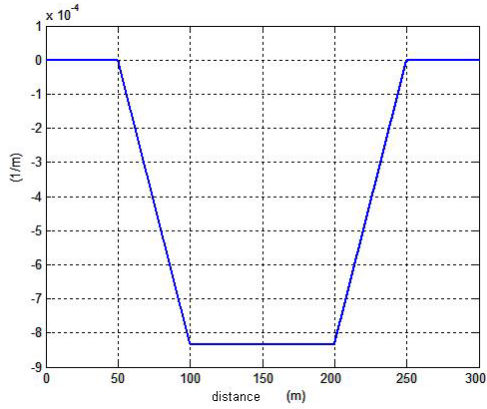

a)

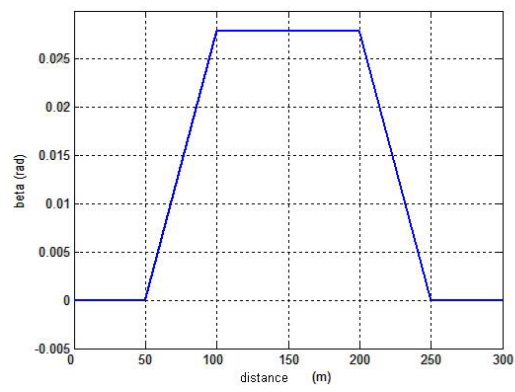

b)

Figure 4: Test features: a) track curvature, b) cant angle $(R=1200 \mathrm{~m}$, $\alpha_{p}=1 / 40 \mathrm{rad}, \beta=40 / 1435 \mathrm{rad}, V=30 \mathrm{~m} / \mathrm{s}$ ).

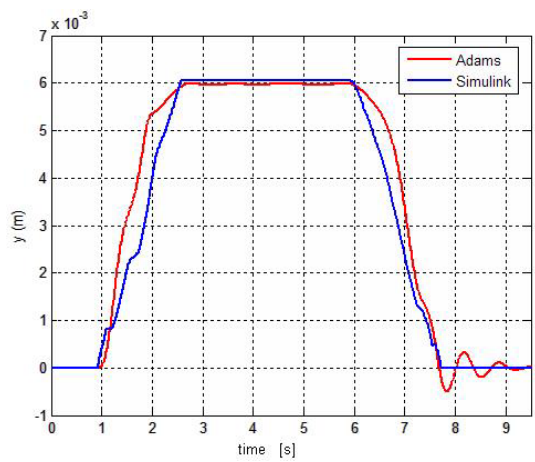

a)

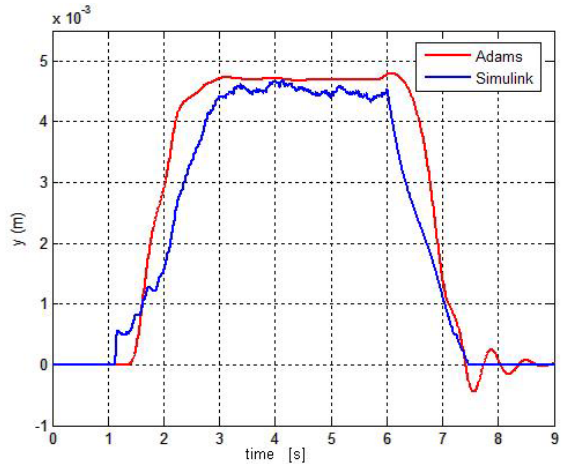

b)

Figure 5: Wheelsets center of mass displacement: comparison between results obtained with Adams and Simulink models: a) First wheelset (front bogie, front wheelset), b) second wheelset (front bogie, rear wheelset).

The simulation results allow one to conclude that the Simulink and Adams multibody models give substantially comparable results. The time history analysis shows that the Simulink model is generally characterized by a higher transient damping, due to the different method for the calculation of the normal component of the contact force (in the Simulink model a nonlinear elasto-viscous approach is implemented). 
Table 1: Hunting frequencies, comparison between the results obtained through Adams and Simulink models.

\begin{tabular}{|l|l|l|}
\hline \multirow{2}{*}{ Speed $(\mathrm{m} / \mathrm{s})$} & \multicolumn{2}{|l|}{ Hunting Frequencies (Hertz) } \\
\cline { 2 - 3 } & ADAMS & SIMULINK \\
\hline 25 & 1.7 & 1.6 \\
\hline 40 & 2.5 & 2.6 \\
\hline 55 & 3.3 & 3.5 \\
\hline 70 & 4.6 & 4.4 \\
\hline
\end{tabular}

\section{Conclusions}

The objective of the work presented in the paper is the development of a numerically efficient model describing the three-dimensional dynamics of a railway vehicle. The challenge is the realization of a real time implementation, to be used for example to control an HIL test rig. In this type of test rig the reliability of the realized tests depends on the accuracy of the simulation of the 'virtual' environment, on the other hand the real time constraint imposes limitations on the complexity of the numerical models and on their integration times. The development of such models has then to find a compromise between accuracy and efficiency.

This paper summarizes the main features of a railway vehicle multibody model realized in the Matlab-Simulink environment. The model was tuned in order to reproduce the dynamical behavior of a benchmark railway vehicle. The results of the simulations carried out with this model are compared with those obtained with the Adams Rail multibody model of the same vehicle. The comparison has shown a substantially good agreement between the models and the relative errors are acceptable. It has to be highlighted that the models evaluate the local deformation of the wheel and the rail in the contact zone by means of two different approaches. With respect to the existing and available railway multibody models, its features are then a more detailed modeling of the wheel/rail contact problems and the possibility to easily obtain an executable implementation that could run in real time conditions. In the developed model the aspects relative to the contact point definition and contact force calculation has been carefully investigated since they have a significant effect on the vehicle dynamics.

\section{References}

[1] Wolfgang Rulka, Eli Pankiewicz, MBS Approach to Generate Equations of Motions for HiL-Simulations in Vehicle Dynamics Multibody System Dynamics (2005) n.14, pp 367-386. 
[2] L. Pugi, M. Malvezzi, A. Tarasconi, A. Palazzolo, G. Cocci, M. Violani, "HIL Simulation of WSP Systems on MI-6 Test Rig", Proceedings of the XIX IAVSD 2005 - International Vehicle System Association, Milano 29 agosto-2 September 2005. Vehicle system Dynamics, Vol. 44, Supplement 2006 pp.843-852 ISBN 978-0-415-43616-8.

[3] A. A. Shabana, M. Tobaa, H. Sugiyama, K. E. Zaazaa, On the Computer Formulation of the Wheel/Rail Contact Problem, Nonlinear Dynamics, Springer 2005, vol. 40, pp. 169-193.

[4] Pombo, J. Ambrosio, Dynamics analysis of a railway vehicle in real operation conditions using a new wheel-rail contact detection model, "Int. J. of Vehicle Systems Modelling and Testing”, 1, 2005

[5] De Pater, A. D., 'The geometric contact between track and wheelset', Vehicle System Dynamics 17, 1988, 127-140.

[6] L. Baeza, A. Roda, J. Carballeira, E. Giner, Railway Train-Track Dynamics for Wheelflats with Improved Contact Models, Nonlinear Dynamics, Springer 2006, vol. 45, pp. 385-397.

[7] T. G. Kolda, R. M. Lewis, V. Torczon, Optimization by direct search: new perspectives on some classical and modern methods, SIAM Review, Vol. 45, N. 3 (2003), pp. 385-482.

[8] M. W. J. C. Lagarias, J. A. reeds, P.E. Wright, convergence properties of the Nelder Mead simplex method in low dimensions, SIAM Journal on Optimization, No. 9 (1998), pp. 112-147.

[9] R. V. Dukkipati, J. R. Amyot, Computer aided simulation in railway dynamics, Marcel Dekker, 1988W. Schiehlen. Multibody system dynamics: Roots and perspectives. Multibody System Dynamics, 1, 149-188, 1997.

[10] J.J. Kalker, Three-Dimensional Elastic Bodies in Rolling Contact, Kluwer Academic Publishers, 1990.

[11] E.A.H. Vollebregt, J.J. Kalker, G. Wang, " CONTACT 93 Users Manual“, VORtech Computing, Industrial and Scientific Computing, July 1992, revised March 1994.

[12] K.L. Johnson, Contact mechanics, Cambridge University Press, 1985.

[13] P.J. Vermeulen, K.L. Johnson, Contact of Nonspherical Elastic Bodies Transmitting Tangential Forces, Journal of Applied Mechanics, Transactions of the ASME, June 1964.

[14] Iwnicki S., 'The Manchester Benchmarks for Rail Vehicle Simulators' Swets \& Zeitlinger B.V. Lisse 1999, (ISBN 9026515510 ) 